\title{
SISTEMATIZAÇÃO DO DIMENSIONAMENTO TÉCNICO E ECONÔMICO DE SISTEMAS FOTOVOLTAICOS ISOLADOS POR MEIO DE PROGRAMA COMPUTACIONAL ${ }^{1}$
}

\author{
JOSÉ A. MARINI ${ }^{2}$, LUIZ A. ROSSI ${ }^{3}$
}

RESUMO: Uma das principais questões referentes à energia solar é como compará-la, técnica e economicamente, com outras fontes de energia, tanto alternativas quanto com as convencionais (como a rede elétrica). O propósito deste trabalho foi desenvolver um programa computacional que reúne os principais dados técnicos e econômicos, para identificar, por meio de métodos de análise microeconômica, a viabilidade comercial no dimensionamento de sistemas fotovoltaicos, além de considerar os benefícios provenientes da própria geração energética. Na análise microeconômica da energia solar, foram identificados os custos da energia proveniente da rede convencional durante o período de vida útil dos componentes do sistema gerador de eletricidade fotovoltaica, pelo estudo dos custos de investimentos iniciais e manutenção do sistema. Para comparação com as fontes convencionais - rede elétrica e grupo diesel - foram usados três cenários de custos de painéis fotovoltaicos e dois para o fator de disponibilidade do grupo diesel. Pelos resultados, verifica-se que, quanto mais baixo o custo dos painéis e mais distante o local situar-se da rede elétrica, o sistema fotovoltaico torna-se a opção mais vantajosa.

PALAVRAS-CHAVE: energia solar, programa computacional, sistema fotovoltaico.

\section{SYSTEMATIZATION OF THE TECHNICAL AND ECONOMIC SIZING OF ISOLATED PHOTOVOLTAIC SYSTEMS THROUGH SPECIFIC SOFTWARE}

\begin{abstract}
One of the main referring subjects to the solar energy is how to compare it economically with other sources of energy, as much alternatives as with conventionals (like the electric grid). The purpose of this work was to develop a software which congregates the technical and economic main data to identify, through methods of microeconomic analysis, the commercial viability in the sizing of photovoltaic systems, besides considering the benefits proceeding from the proper energy generation. Considering the period of useful life of the components of the generation system of photovoltaic electricity, the costs of the energy proceeding from the conventional grid had been identified. For the comparison of the conventional sources, electric grid and diesel generation, three scenes of costs of photovoltaic panels and two for the factor of availability of diesel generation had been used. The results have shown that if the cost of the panels is low and the place of installation is more distant of the electric grid, the photovoltaic system becomes the best option.
\end{abstract}

KEYWORDS: solar energy, software, photovoltaic system.

\section{INTRODUÇÃO}

O suprimento energético a comunidades rurais isoladas e a áreas remotas tem sido um constante desafio, principalmente nos países subdesenvolvidos ou em desenvolvimento, devido às enormes disparidades econômicas e sociais e, muitas vezes, às dimensões geográficas desses países.

Há algum tempo, os sistemas de suprimento de energia alternativa descentralizados, utilizando recursos renováveis, têm sido empregados no atendimento a comunidades mais isoladas. Vários tipos desses sistemas têm sido estudados, mostrando-se economicamente viáveis e/ou tecnicamente

\footnotetext{
${ }^{1}$ Extraído da dissertação de mestrado do primeiro autor.

${ }^{2}$ Mestrando em Engenharia Agrícola, Faculdade de Engenharia Agrícola, Universidade Estadual de Campinas, Campinas - SP.

${ }^{3}$ Prof. Doutor, Faculdade de Engenharia Agrícola, Universidade Estadual de Campinas, Campinas - SP.

Recebido pelo Conselho Editorial em: 14-8-2002

Aprovado pelo Conselho Editorial em: 28-2-2005
}

Eng. Agríc., Jaboticabal, v.25, n.1, p.67-75, jan./abr. 2005 
factíveis. O avanço da tecnologia, principalmente nas áreas solar e eólica, vem criando opções para a geração de eletricidade de forma não poluente (PALZ, 1981).

Para saber se a energia solar é um investimento economicamente viável, é necessário recorrer ao uso de modelos de análise que avaliem tanto os custos do investimento quanto os benefícios decorrentes do mesmo (COSTA et al., 2000 e FADIGAS, 1993).

Quando os custos de investimento são elevados e os benefícios resultantes são distribuídos em função do tempo - como ocorre na maioria das aplicações com energia solar - é conveniente fazer uma análise econômica sobre a vida útil do equipamento a ser utilizado antes de se investir (RUEGG \& SAV, 1980).

Dentre os métodos econômicos disponíveis, podem-se citar os mais comuns, que são: o da taxa de retorno, o da relação custo/benefício, o do tempo de retorno e o do custo da vida útil. Os métodos de avaliação econômica de sistemas energéticos diferem essencialmente na maneira em que relacionam custos e benefícios que, mesmo ligados entre si, não são necessariamente excludentes, pois tratam de tipos diferentes de decisões de investimentos. Assim, para alguns tipos de decisões, a escolha de um método é mais importante do que a de outro (HIRSCHEFELD, 1996).

Por alocar um orçamento limitado, entre várias oportunidades de investimentos disponíveis, o método que relaciona custo/benefício ou o método da taxa de retorno são os mais recomendados porque podem fornecer ao usuário melhor seleção dos projetos, que proporcionarão um retorno total maior para um determinado nível de investimento.

O objetivo do trabalho foi desenvolver um programa computacional que reunisse os principais dados técnicos e econômicos, para identificar, através de métodos de análise microeconômica, a viabilidade comercial no dimensionamento de sistemas fotovoltaicos, considerando os benefícios provenientes da própria geração energética.

\section{MATERIAL E MÉTODOS}

Programas computacionais que realizam análises conjuntas de aspectos técnicos e econômicos são intensamente empregados na área de geração, transmissão e distribuição de energia elétrica.

Assim, o programa computacional desenvolvido neste trabalho apresenta-se bastante adequado para análise de sistemas de suprimento de energia elétrica em comunidades isoladas, que são aquelas muito distantes da rede elétrica ou separadas por alguma formação geográfica, a qual impede a chegada da rede elétrica em condições técnicas e econômicas favoráveis.

Junto com o dimensionamento do sistema fotovoltaico, baseado nas principais cargas elétricas previstas em qualquer comunidade isolada, o programa computacional desenvolvido fornece uma análise econômica, indicando a relação custo/benefício e o valor do capital que poderia ser economizado com a instalação, por meio do índice de benefício líquido.

$\mathrm{Na}$ análise econômica, primeiramente foi definido o custo inicial de investimento de cada alternativa, o qual representa o capital necessário para construir uma alternativa de geração. Considerando que os investimentos são concentrados no ano de início de operação da alternativa, o custo de investimento de uma alternativa é dado pela somatória dos investimentos individuais de cada componente do sistema que, para o sistema fotovoltaico, são: sistema gerador, bateria e inversor. Para a rede elétrica, o investimento inicial é calculado com base nos custos das linhas e componentes associados (transformador, postes, chaves de proteção e outros) e os componentes do sistema grupogerador são: o próprio grupo-gerador, carregador de bateria, bateria e inversor.

Segundo ROBERTS (1990) e BOILY et al. (1998), o investimento inicial de cada item de custo, para cada alternativa a ser analisada, é calculado pela eq.(1):

$$
\mathrm{INV}_{\mathrm{i}}=\mathrm{CN}_{\mathrm{i}} \mathrm{PI}_{\mathrm{i}}
$$


em que,

$\mathrm{INV}_{\mathrm{i}}$ - investimento inicial do componente $i, \mathrm{US} \$$;

$\mathrm{CN}_{\mathrm{i}}$ - custo unitário do componente $i, \mathrm{US} \$ \mathrm{~kW}^{-1}$, e

$\mathrm{PI}_{\mathrm{i}}$ - potência instalada do componente $i, \mathrm{~kW}$.

Especificamente para a rede elétrica, o investimento inicial é calculado pela eq.(2):

INVRE $=$ CNKM DST

em que,

INVRE - investimento inicial na rede elétrica, US\$;

CNKM - custo por $\mathrm{km}$ das linhas e equipamentos instalados, US\$ $\mathrm{km}^{-1}$, e

DST - comprimento da rede, $\mathrm{km}$.

De uma forma geral, para os componentes que possuem vida útil inferior ao horizonte de comparação, faz-se necessário calcular o custo de reposição dos mesmos. Tecnicamente, o horizonte de comparação é igual à maior vida útil entre aquelas dos componentes de custo de uma alternativa. $\mathrm{O}$ valor presente do investimento de cada componente do sistema analisado, considerando sua reposição, é calculado pela eq.(3).

$$
\mathrm{VP}_{\mathrm{i}}=\mathrm{INV}_{\mathrm{i}}\left[1+\sum \frac{1}{(1+\mathrm{x})^{\mathrm{Ni}} \mathrm{r}}\right], \mathrm{r}=0,1,2,3 \ldots\left(\mathrm{N}_{\mathrm{i}} \mathrm{r}\right)<\mathrm{H}
$$

em que

$\mathrm{VP}_{\mathrm{i}}$ - valor presente do investimento do componente $i$ do sistema analisado, US\$;

$\mathrm{INV}_{\mathrm{i}}$ - investimento inicial do componente $i$, US\$;

$\mathrm{x}$ - taxa de desconto de referência ou de atratividade em decimal;

$\mathrm{N}_{\mathrm{i}}$ - vida útil do componente $i$, anos, e

$\mathrm{H}$ - horizonte de comparação, anos.

O custo anual de operação e manutenção é igualmente calculado para todas as alternativas analisadas neste trabalho, pela eq.(4):

$\mathrm{CAOM}=\mathrm{TXOM} \mathrm{INV}_{\mathrm{i}}$

em que,

CAOM - custo anual de operação e manutenção, US\$;

TXOM - taxa porcentual dos custos de operação e manutenção, \%, e

$\mathrm{INV}_{\mathrm{i}}$ - investimento inicial do componente $i$, US\$.

O valor atual dos custos de operação e manutenção é expresso pela eq.(5):

$\mathrm{VAOM}=\mathrm{CAOM} \frac{(1+\mathrm{x})^{H}-1}{x(1+x)^{H}}$

em que,

VAOM - valor atual dos custos anuais de operação e manutenção, US\$.

O custo anual de suprimento do sistema de geração é calculado pela eq.(6).

$\mathrm{CAGE}=\mathrm{CME}$ DE $365+\mathrm{CMP} \mathrm{P}$

em que,

CAGE - custo anual de suprimento pelo sistema de geração, US\$;

$\mathrm{CME}$ - custo marginal de energia, US\$ $\mathrm{kWh}^{-1}$;

DE 365 - necessidade anual de energia do sistema isolado, $\mathrm{kWh}$;

CMP - custo marginal de ponta, US $\$ \mathrm{~kW}^{-1}$, e

$\mathrm{P}$ - necessidade de potência do sistema isolado, $\mathrm{kW}$. 
O valor atual dos custos totais de investimento da alternativa em questão é dado pela somatória do valor atual do investimento de cada componente do sistema, acrescido dos custos indiretos. Sua expressão é dada por (7).

$$
\mathrm{VA}_{\mathrm{t}}=\Sigma \mathrm{VP}_{\mathrm{i}}+\left(\mathrm{TX} \Sigma \mathrm{VP}_{\mathrm{i}}\right)
$$

em que,

$\mathrm{VA}_{\mathrm{t}}$ - valor atual do custo total de investimento da alternativa analisada, US\$, e

TX - taxa de custos indiretos (custos relativos à engenharia, administração e outros), \%.

O valor anual do custo total de investimento pode ser analisado pela eq.(8).

$\mathrm{VACT}=\mathrm{VAt}$ FRC

em que,

VACT - valor anual do custo total de investimento, US\$, e

FRC - Fator de recuperação do capital (adimensional), em que, FRC é dado por:

$$
\mathrm{FRC}=\frac{x(1+x)^{H}}{(1+x)^{H}-1}
$$

O equivalente anual dos custos totais envolvidos em uma alternativa é dado pela eq.(9).

$$
\mathrm{CTAE}=\mathrm{AINV}+\mathrm{VAOM}+\mathrm{VACT}+\mathrm{VASE}
$$

em que,

CTAE - custo total anual equivalente, US\$;

AINV - valor anual do custo total de investimento, US\$;

VAOM - valor anual dos custos de operação e manutenção, US\$;

VACT - valor anual do custo do combustível mais transporte, US\$, e

VASE - valor anual do custo de suprimento pela rede elétrica, US\$.

O custo anual de geração para o sistema fotovoltaico e para o grupo gerador é expresso pela razão entre o custo total anual equivalente e a energia anual gerada. De acordo com FADIGAS (1993) e ROSSI (1995), ele é dado pela eq. (10).

$$
\mathrm{CAG}=\frac{\mathrm{CTAE}}{\text { EG/ano }}
$$

em que,

CAG - custo anual de geração, US\$ $\mathrm{kWh}^{-1}$;

CTAE - custo total anual equivalente, US\$, e

EG/ano - energia anual gerada, $\mathrm{kWh}$.

A energia anual gerada pode ser calculada pela eq. (11)

$\mathrm{EG} / \mathrm{ano}=\mathrm{PI}$ FC DI 8760

em que,

PI - potência instalada, $\mathrm{kW}$;

FC - fator de capacidade, \%;

DI - fator de disponibilidade da alternativa de suprimento, $\%$, e

8760 - número de horas anuais. 
Para a alternativa rede elétrica, o custo anual de geração é determinado como sendo a relação entre o custo anual equivalente e a energia anual solicitada pelo sistema isolado. É dado pela eq.(12).

$$
\text { EGano }=\frac{\text { CTAE }}{\text { CDT } 365 \text { DI }}
$$

em que,

$\mathrm{EG}_{\mathrm{ano}}$ - energia requerida pela carga ou entregue à carga pelo sistema de geração, $\mathrm{kWh}, \mathrm{e}$

CDT - consumo diário de energia pela carga, kWh.

O benefício líquido é expresso como a diferença entre o benefício total, ou seja, o quanto se economizaria em gastos com a tarifa energética e o custo total, ambos traduzidos em valores anuais equivalentes. Para cada alternativa, o benefício líquido é calculado pela eq.(13).

$$
\mathrm{BL}=\mathrm{BE}-\mathrm{CTAE}
$$

em que,

BL - benefício líquido, US\$;

BE - benefício total proveniente da economia em energia elétrica, US\$, em que BE é dado por:

$$
\mathrm{BE}=\mathrm{CDT} 365 \mathrm{TFF}
$$

em que,

TFF - tarifa de fornecimento de energia, US\$ $\mathrm{kWh}^{-1}$.

A relação custo-benefício $(\mathrm{CBF})$ indica o retorno em anos do investimento e é fornecido por:

$\mathrm{CBF}=\mathrm{CTAE} / \mathrm{BE}$

Deve ser lembrado que, embora essa relação indique período de retorno, não se deve usá-la para classificar as alternativas. Isso deve ser feito aplicando-se o método do valor presente líquido ou valor anual dos custos totais, que consideram o fluxo de caixa completo. Ambos os métodos estão presentes nessa análise, e seus resultados saem impressos nos relatórios do programa computacional.

A linguagem computacional Visual Basic 6 (WANG, 2000) foi utilizada para desenvolver a estrutura lógica de funcionamento do programa de dimensionamento técnico e econômico de sistemas fotovoltaicos. Esse programa recebeu o nome comercial de UNIVOLT.

\section{RESULTADOS E DISCUSSÃO}

Pelo apresentado, o programa computacional teve o objetivo de sistematizar o desenvolvimento do projeto de sistemas fotovoltaicos de produção de eletricidade e posterior análise de sua viabilidade econômica frente os custos de expansão da rede elétrica convencional e da instalação de um sistema grupo gerador. O projeto do sistema fotovoltaico é sempre desenvolvido baseado nas cargas elétricas presentes no local onde se deseja implantar um sistema de geração de energia elétrica e seus respectivos consumos energéticos. Posteriormente, é fornecida uma análise da viabilidade econômica de implantação do projeto e, se for o caso, do tempo de retorno do capital investido, baseado nas economias com o uso da rede elétrica.

A validação do programa foi feita comparando seus resultados com os de outros projetos já instalados. Os dados usados foram obtidos de FADIGAS (1993) e BOILY et al. (1998), e todos os preços e valores econômicos envolvidos nos cálculos referem-se ao ano de 2002. 
A tela do programa, na qual são solicitados para serem inseridos os principais dados técnicos e econômicos, está ilustrada na Figura 1. As telas que mostram os resultados finais do dimensionamento e da análise econômica são mostradas, respectivamente, pelas Figuras 2 e 3.

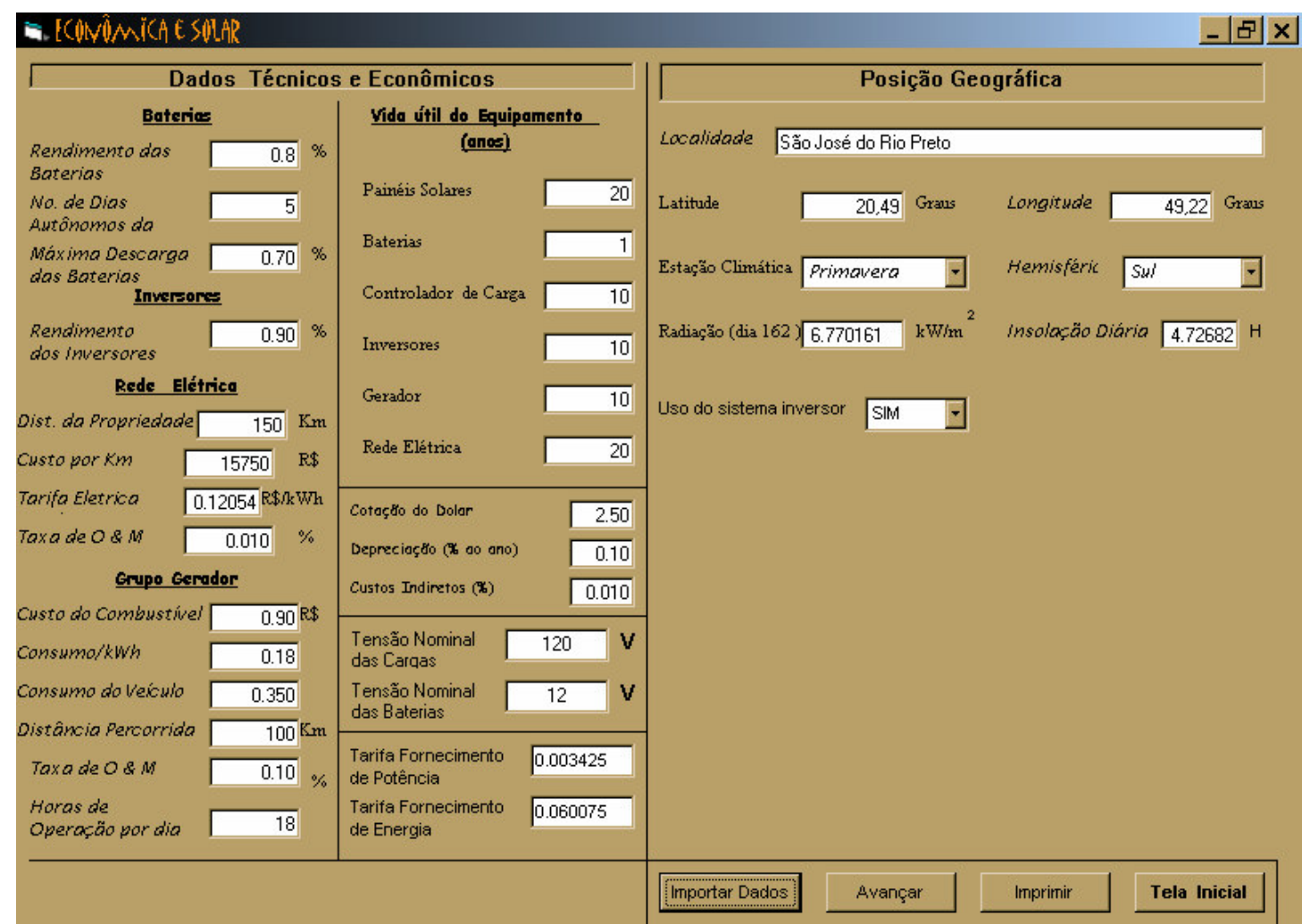

FIGURA 1. Entrada de dados econômicos e técnicos.

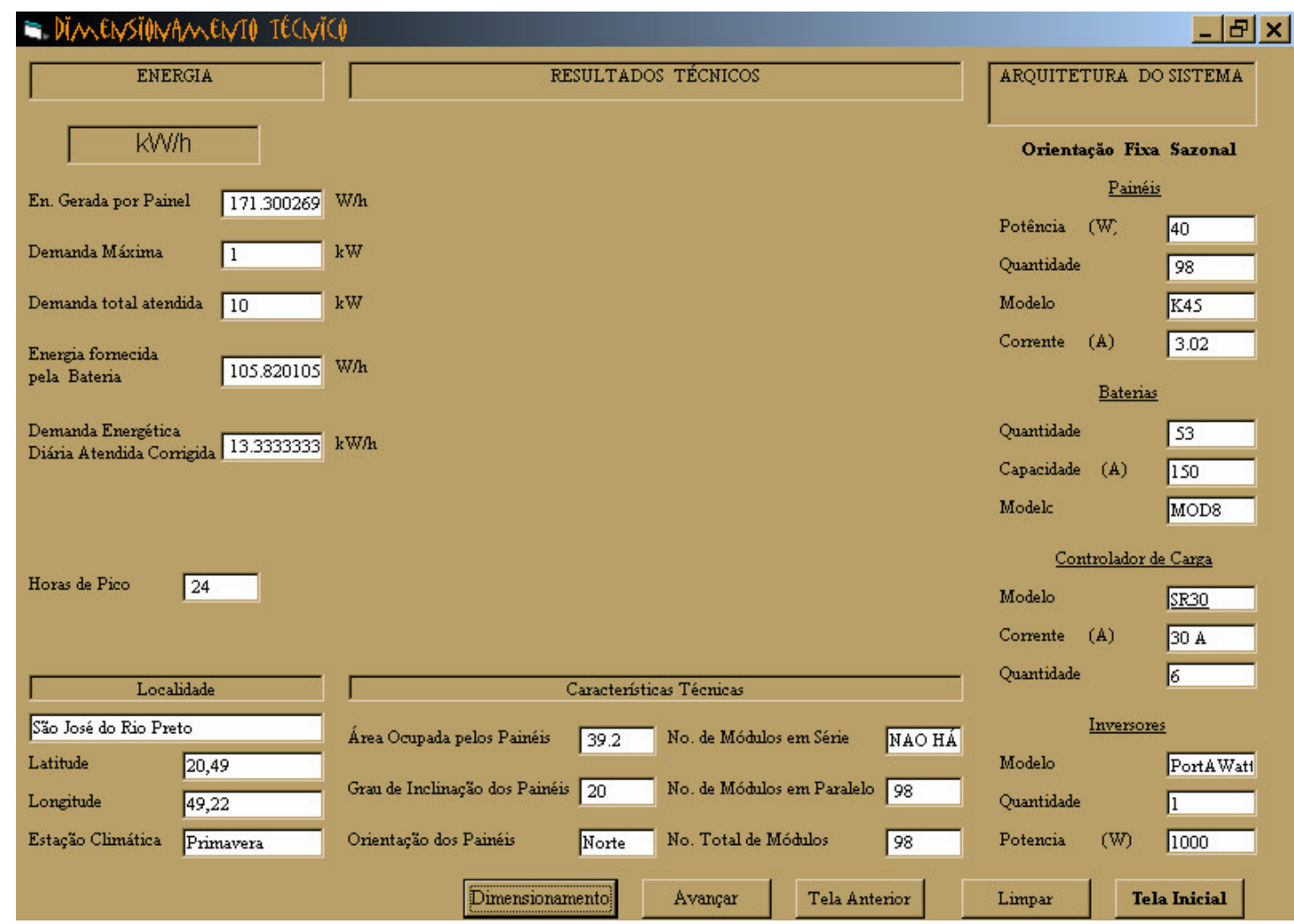

FIGURA 2. Apresentação do dimensionamento do sistema. 


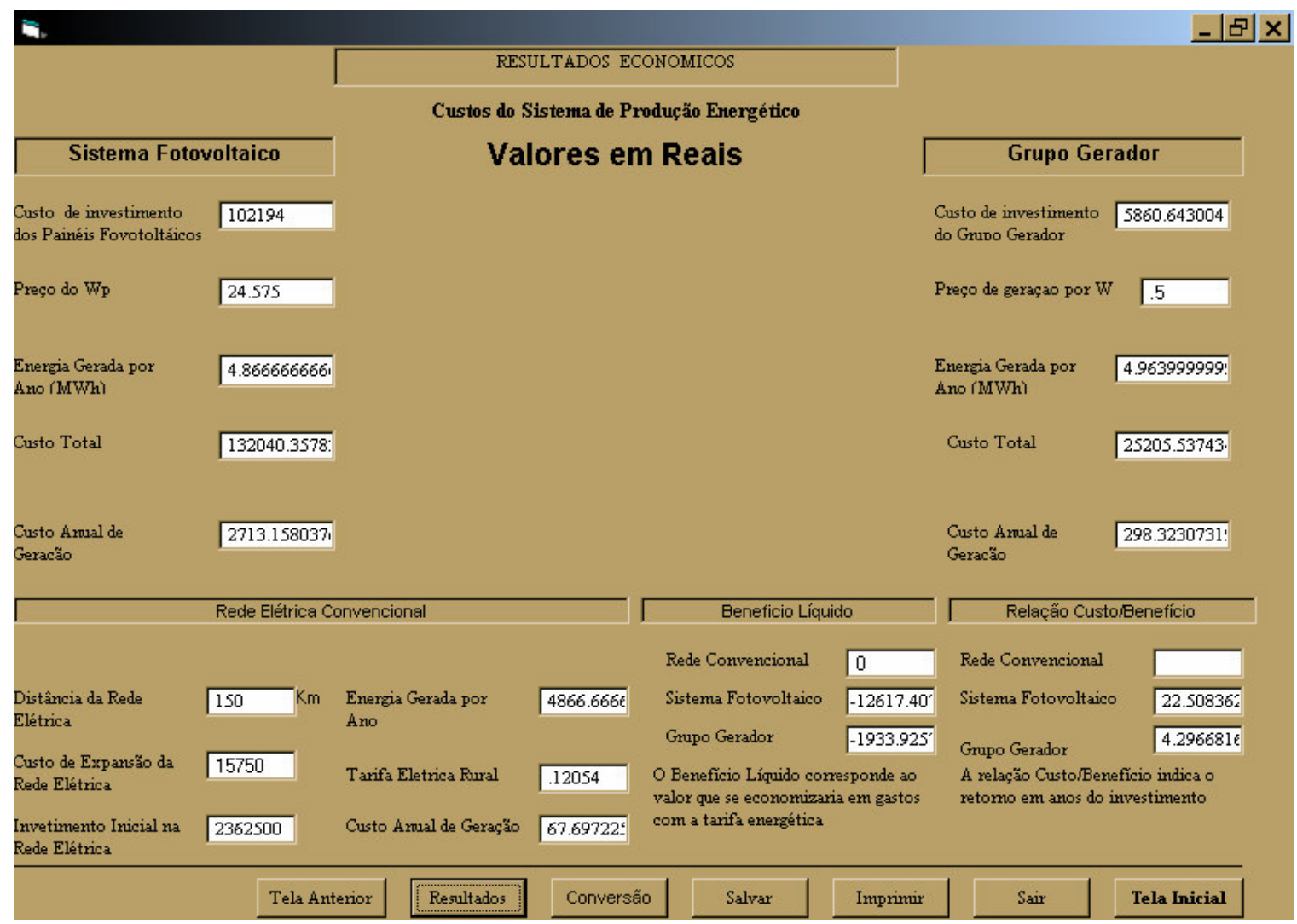

FIGURA 3. Apresentação dos resultados econômicos do sistema dimensionado.

No Brasil, o consumo diário médio de energia elétrica em uma típica propriedade rural (ROSSI, 1995 ) está em torno de $45 \mathrm{kWh}$, com picos máximos de $5 \mathrm{~kW}$. Com essa característica de atendimento, o programa forneceu a análise econômica ilustrada pela Tabela 1. Esse exemplo de cálculo baseou-se no valor do $\mathrm{W}_{\text {pico }}\left(\mathrm{W}_{\mathrm{p}}\right)$ de $\mathrm{U} \$ 9,00$, que é um dos cenários considerados para o custo de investimento do sistema fotovoltaico.

TABELA 1. Resultados econômicos fornecidos pelo Programa Univolt para análise econômica com demanda diária de $45 \mathrm{kWh}$ e pico de $5 \mathrm{~kW}$.

\begin{tabular}{lrr}
\hline \multirow{2}{*}{ Parâmetros } & \multicolumn{2}{c}{ Valor } \\
\cline { 2 - 3 } & \multicolumn{1}{c}{ Real } & \multicolumn{1}{c}{ Dólar } \\
\hline Fator de capacidade (\%) & 23,54 & 23,54 \\
Custo inicial de instalação & $61.220,00$ & $24.488,00$ \\
Valor presente dos custos totais & $69.114,07$ & $27.645,63$ \\
Custo anual equivalente & $8.114,10$ & $3.245,64$ \\
Custo anual de geração (valor/MWh) & 494,01 & 197,60 \\
Índice custo/benefício & 4,09 & 3,47 \\
Benefício líquido & $-6.134,23$ & $-2.311,55$ \\
Energia anual gerada (MWh) & 16,425 & 16.425 \\
\hline
\end{tabular}

Como pode ser notado na Tabela 1, o programa computacional forneceu resultados técnicos e econômicos que conduziram a uma consistente tomada de decisão com base nos critérios usualmente empregados para tal fim. Podem ser feitas análises de sensibilidade usando-se vários parâmetros técnicos ou econômicos, tais como, demanda a ser atendida, taxa de atratividade, nível de investimento, etc. 
Usando-se o equacionamento apresentado na metodologia, para a fonte solar fotovoltaica, grupo gerador diesel e rede elétrica, para ser atendida a mesma demanda acima, obtiveram-se os resultados comparativos mostrados na Figura 4.

Custo de Geração x Distância

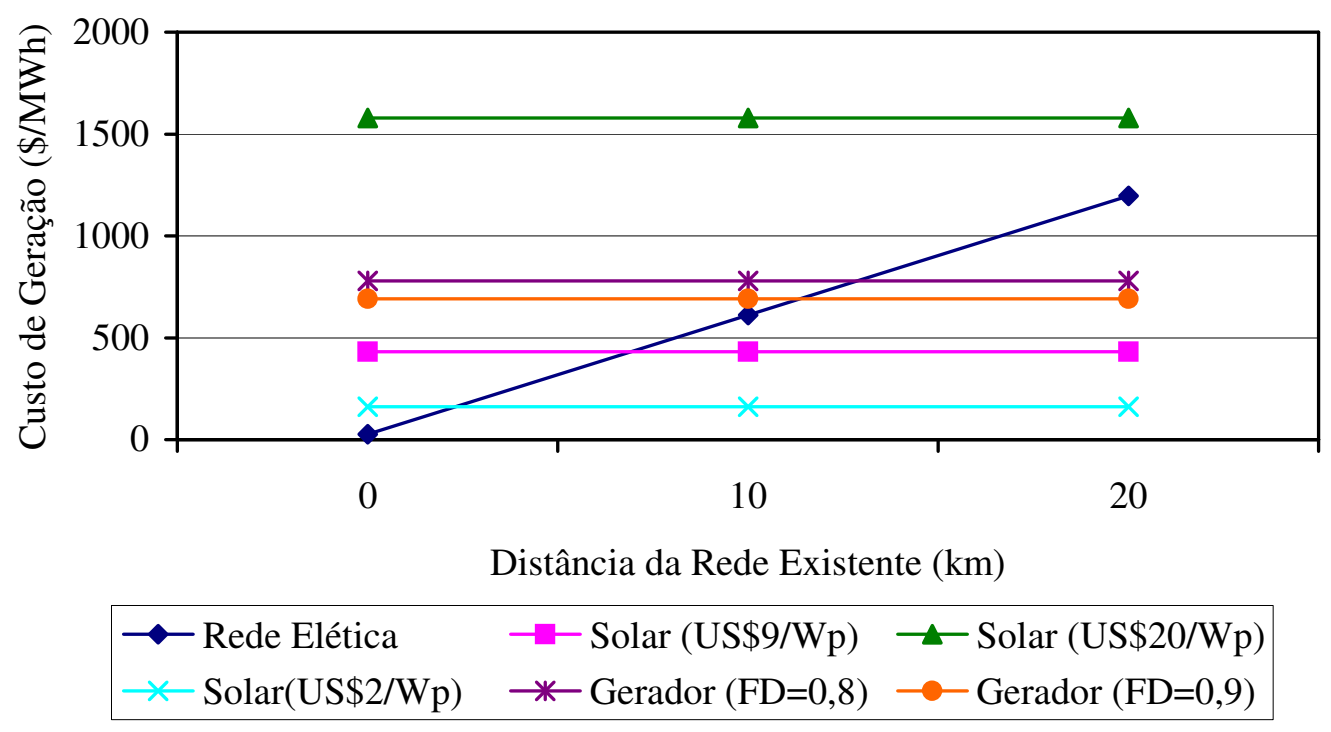

FIGURA 4. Resultados econômicos com diferentes valores de Wp para painéis fotovoltaicos e dois fatores de disponibilidade de um grupo gerador comparativamente com a distância em $\mathrm{km}$ da propriedade em relação à rede elétrica convencional já existente, no suprimento de demanda diária de $45 \mathrm{kWh}$.

Nota-se que, pelos cenários apresentados, a fonte solar fotovoltaica torna-se competitiva apenas a partir de uma certa distância do ponto de origem da rede elétrica; mesmo assim, para o melhor cenário de preço de painel fotovoltaico. Por esse exemplo de projeto de dimensionamento e simulação do fornecimento de energia elétrica com outras fontes, vê-se que podem ser realizadas várias possibilidades de análises de sensibilidade de parâmetros ou variáveis importantes, conduzindo a soluções específicas para locais com características distintas.

\section{CONCLUSÕES}

Após a validação do programa, constatou-se que a viabilidade econômica de projetos fotovoltaicos só ocorre quando não há qualquer possibilidade de expansão da rede elétrica convencional até a propriedade, tendo em vista, principalmente, os altos custos dos painéis fotovoltaicos, principal componente do sistema de conversão de luz solar em energia elétrica.

Quanto à funcionalidade, como ficou evidente nas duas simulações apresentadas, o programa pode vir a ser uma ferramenta útil no projeto e análise econômica de sistemas fotovoltaicos no Brasil.

\section{REFERÊNCIAS}

BOILY, R.; SAWADOGO, A.; ROSSI, L.A. Projeto de sistemas fotovoltaicos para comunidades rurais. In: CONGRESSO BRASILEIRO DE ENGENHARIA AGRÍCOLA, 27., 1998, Poços de Caldas. Anais... Lavras: Sociedade Brasileira de Engenharia Agrícola, 1998. v.3, p.392-3. 
COSTA, H.S.; COSTA, R.A.L.; ECK, M. Análise econômica comparativa da eletrificação rural comercial e fotovoltaica. In: ENCONTRO DE ENERGIA NO MEIO RURAL, 3., 2000, Campinas. Anais... Campinas: UNICAMP, 2000. 1 CD.

FADIGAS, E. Dimensionamento de fontes fotovoltaicas e eólicas com base no índice de perda de suprimento e sua aplicação para o atendimento a localidades isoladas. 1993. $163 \mathrm{f}$. Dissertação (Mestrado em Planejamento Energético) - Escola Politécnica, Universidade de São Paulo, São Paulo, 1993.

PALZ, W. Energia solar e fontes alternativas. São Paulo: Hemus, 1981. 358 p.

HIRSCHEFELD, H. Engenharia econômica e análise de custo. 6.ed. São Paulo: Atlas, 1996. 407 p.

ROBERTS, S. Solar electricity. $2^{\text {nd }}$ ed. New York: Prentice Hall, 1990. 347 p.

ROSSI, L.A. Modelo avançado para planejamento de sistemas energéticos integrados usando recursos renováveis. 1995. 180 f. Tese (Doutorado em Planejamento Energético) - Escola Politécnica, Universidade de São Paulo, São Paulo, 1995.

RUEGG, R.; SAV, G.T. The microeconomics of solar energy. $3^{\text {th }}$ ed. Washington: National Bureau of Standards, $1980.189 \mathrm{p}$.

WANG, W. Visual Basic 6 for Dummies. Petrópolis: Campus, 2000. 532 p. 